\author{
Classification \\ Physics Abstracts \\ $8.810-8.820-8.550-8.540$
}

\title{
LOW TEMPERATURE OPTICAL STUDY OF MANGANOUS CHLORIDE
}

\author{
M. REGIS and Y. FARGE \\ Laboratoire de Physique des Solides $\left({ }^{*}\right)$ \\ Université Paris-Sud, 91405 Orsay, France
}

(Reçu le 21 mars 1975, révisé le 5 janvier 1976, accepté le 19 janvier 1976)

\begin{abstract}
Résumé. - Dans cet article, le spectre optique visible de $\mathrm{MnCl}_{2}$ est mesuré à basse température $(4,18 \mathrm{~K})$. On calcule le dichroïsme circulaire magnétique (DCM) pour la transition optique ${ }^{6} \mathrm{~A}_{1 \mathrm{~g}}\left({ }^{6} \mathrm{~S}\right) \rightarrow{ }^{4} \mathrm{~A}_{1 \mathrm{~g}}\left({ }^{4} \mathrm{G}\right),{ }^{4} \mathrm{Eg}\left({ }^{4} \mathrm{G}\right)$ dans le cas où le champ magnétique appliqué est parallèle à l'axe c du cristal. On expose les résultats expérimentaux de DCM et de dichroïsme circulaire en champ nul, qui permettent de tracer le diagramme de phase magnétique entre $4,18 \mathrm{~K}$ et $1,6 \mathrm{~K}$ pour des champs de 0 à $25 \mathrm{kG}$. Un signal de dichroïsme linéaire est aussi observé dans les deux phases ordonnées. L'ordre magnétique est ensuite discuté d'après ces nouvelles données expérimentales.
\end{abstract}

Abstract. - The low temperature absorption spectrum of $\mathrm{MnCl}_{2}$ is reported. A calculation of the expected magnetic circular dichroïsm and its correlation with magnetization is performed for the optical transition ${ }^{6} \mathrm{~A}_{1 \mathrm{~g}}\left({ }^{6} \mathrm{~S}\right) \rightarrow{ }^{4} \mathrm{~A}_{1 \mathrm{~g}}\left({ }^{4} \mathrm{G}\right),{ }^{4} \mathrm{Eg}\left({ }^{4} \mathrm{G}\right)$ at $23557 \mathrm{~cm}^{-1}$. The experimental results of $M C D$ and $C D$ in zero field are reported and the magnetic phase diagram is plotted. A linear dichroïsm is also observed in the two ordered phases. The magnetic order is discussed in view of these new data.

1. Introduction. - It has been already pointed out that, in a lot of transparent magnetic materials, the behaviour of the optical spectrum is related to the magnetic properties and magnetic phase transitions [1].

Schnatterly and Fontana have shown that the oscillator strength of the transition at $\lambda=4270 \AA$ in $\mathrm{FeCl}_{2}$ is related to the nearest-neighbour spin correlation function, and they have also verified a linear dependence between the magnetic circular dichroic signal (MCD) and the magnetization in the antiferromagnetic phase, for the infra-red optical transition. This fact has been used by Griffin and Schnatterly to measure the critical exponents at the tricritical point [2].

Moreover, optical studies of magnon sidebands by polarization techniques and Raman scattering give information on the magnon density of states [3]. On the other hand, linear magnetic birefringence measurements permit one to measure the magnetic energy and the magnetic specific heat [4]. Finally, MCD measurements can also be used for a determination of magnetic phase diagrams, and the effective magnetic splitting factors for many optical transitions [5].

In this paper, we report some experiments performed on $\mathrm{MnCl}_{2}$, by optical absorption and $\mathrm{MCD}$ technique, in a temperature range from 1.5 to $4.18 \mathrm{~K}$. This mate-

(*) Laboratoire associé au C.N.R.S. rial, in spite of its very complex magnetic structure, was chosen because it presents two magnetic transitions, in a range of temperature where optical transitions can be studied with the best precision.

2. Structure and properties of $\mathbf{M n C l}_{2}$. - Anhydrous $\mathrm{MnCl}_{2}$ is a pink lamellar crystal, and crystallises in the rhombohedral or pseudo-hexagonal structure of $\mathrm{CdCl}_{2}$ (space group $\mathrm{D}_{3 \mathrm{~d}}$ ). Hexagonal layers of metal atoms are separated by two layers of chlorine atoms. The crystal field $D_{3 \mathrm{~d}}$ on the manganese ions, is only slightly distorted from the cubic symmetry $\mathrm{O}_{\mathrm{h}}$.

Both the specific heat [6] and the magnetic susceptibility [7] exhibit singularities at low temperature. The neutron diffraction investigations of the magnetic structure of $\mathrm{MnCl}_{2}$, performed by Wilkinson et al. [8] at temperatures down to $1.25 \mathrm{~K}$, show two magnetic phase transitions. One takes place at $1.96 \mathrm{~K}$, between the high temperature paramagnetic phase and an antiferromagnetic phase, called phase I. The second transition takes place around $1.81 \mathrm{~K}$ and results from a reorientation of the magnetic moments to a second type of antiferromagnetic structure (phase II). In both of these antiferromagnetic structures, the magnetic moments are assumed to lie nearly in the hexagonal planes, parallel to one of the three a axes. The model proposed for the magnetic structure consists of ferromagnetic planes, at $57^{\circ}$ to the $\mathrm{c}$ axis, each plane being antiferromagnetically coupled to the next one. 


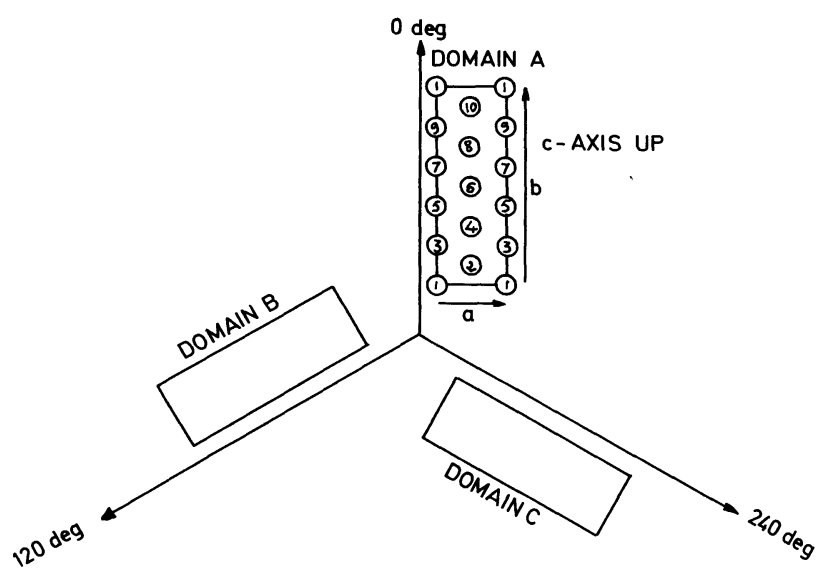

a)

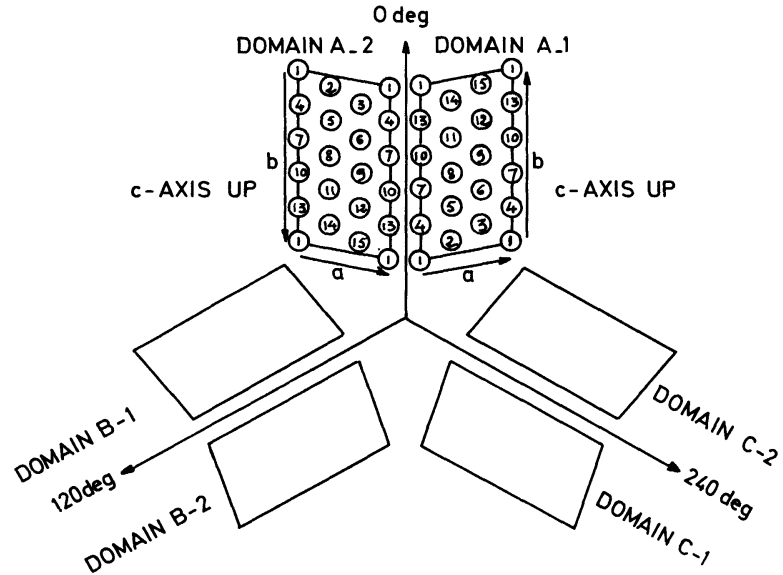

b)

FIG. 1. - Antiferromagnetic domain pattern and magnetic unit cell of $\mathrm{MnCl}_{2}$. a) Antiferromagnetic phase I (between 1.81 and

In phase I, the proposed magnetic unit cell contains ten manganese ions in the base plane and six layers of manganese ions along the $\mathbf{c}$ axis. This unit cell has orthorhombic axes, and this structure can grow in domains along each of the three axes of the basal plane of the chemical hexagonal unit cell. In this temperature range, between 1.81 and $1.96 \mathrm{~K}$, the magnetic lattice is not well ordered, and the coherent scattered intensities are small. The neutron diffraction experiments also reveal the presence of magnetic domains in both states I and II, but, unlike the case of $\mathrm{MnBr}_{2}$, it is not possible to stabilize a single domain in zero field at $1.84 \mathrm{~K}$. This effect is associated with the fact that only a very small degree of long range magnetic order exists in phase I at this temperature, only $0.12 \mathrm{~K}$ below the Néel temperature $(1.96 \mathrm{~K})$.

The proposed magnetic unit cell for phase II is more complex. It consists of 15 manganese ions in the basal plane, and includes 6 layers of manganese ions along the $\mathbf{c}$ axis.

The studies of magnetic reflections as a function of temperature show that a considerable amount of short range order remains above the Néel tempe-
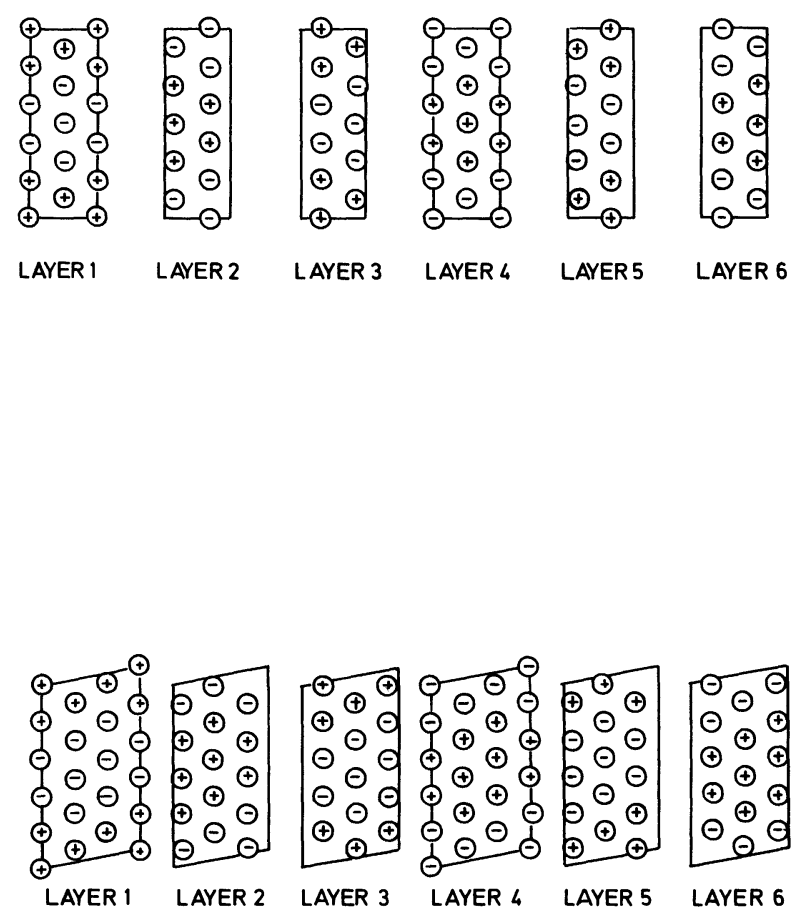

$1.96 \mathrm{~K})$; b) Antiferromagnetic phase II (below $1.81 \mathrm{~K}$ ) (from Wilkinson et al. (8)).

rature. A sligh variation of the Néel temperature after applying a magnetic field is also observed.

The magnetic susceptibilities $\chi_{\|}$and $\chi_{\perp}$ were measured by Murray and Roberts [7]. $\chi_{\|}$does not exhibit the usual maximum in the transition region. $\chi_{\perp}$ does not have a constant value in the ordered phase. In spite of these irregularities, they have concluded that the transition at $1.96 \mathrm{~K}$ is from a paramagnetic to an antiferromagnetic state. They have ruled out the possibility that this transition is ferromagnetic in character because the susceptibility was found to be virtually field independent and no evidence of magnetic hysteresis was observed.

More recent experiments by Giauque et al. [7] and T. Watanabe [7] are not in good agreement with those of Murray and Roberts.

Heat capacity measurements were performed by Murray [6], and more recently by Butera and Giauque [6], in the presence of a magnetic field parallel to one of the a axes in the hexagonal plane. In zero field, the specific heat presents two peaks, located at $1.81 \mathrm{~K}$ and $1.96 \mathrm{~K}$. From these measurements, it was shown that approximately $\frac{2}{3}$ of the total spin entropy has 
been reached in the transition region, so that $\frac{1}{3}$ of the entropy remains to be gained above the transition temperature, through short range order in the spin system, in agreement with the neutron diffraction experiments.

In this paper, after a description of the experimental apparatus (3), the low temperature absorption spectrum is reported (4); a calculation of the expected dichroism and its correlation with magnetization is then performed; the experimental results : $C D$ in zero field, MCD giving the perpendicular magnetization and the magnetic phase diagram are also reported (5). In section 6 are reported the data of MCD when the c axis of the crystal is tilted at a $45^{\circ}$ angle to the light beam and the magnetic field. In section 7 , the magnetic order is discussed.

3. Experimental techniques. - The absorption spectra were performed in a Cary 14 spectrophotometer. The sample was cooled at liquid helium temperature in an immersion cryostat. Temperatures below $4.18 \mathrm{~K}$ were reached by pumping on the helium bath (to $1.5 \mathrm{~K}$ ). The temperature controllers were $10 \Omega$ and $100 \Omega$ Allen Bradley carbon resistors and also a manometer measuring the residual pressure in the helium bath. The magnetic field was produced by a small superconducting solenoid giving fields up to $30 \mathrm{kG}$. In MCD measurements, the directions of the applied magnetic field and of the light beam were parallel. The c axis of the $\mathrm{MnCl}_{2}$ sample was either parallel or inclined at an angle of $45^{\circ}$ to this direction. MCD experiments were made using the polarization modulation technique of Jasperson and Schnatterly [8] with an electro-optic modulator, and an automatic adjustment of the voltage applied to the dynode chain of the photomultiplier to maintain the average output current constant, independent of the light intensity reaching the photomultiplier. In MCD measurements, we used a $1 \mathrm{~m}$ Jarrel-Ash monochromator, with reciprocal dispersion of $8 \AA / \mathrm{mm}$.

The $\mathrm{MnCl}_{2}$ monocrystalline samples were grown by D. Legrand (Département de Physique, Commissariat à l'Energie Atomique, Saclay, France). They were cleaved in thin plates (typically 0.2 to $0.8 \mathrm{~mm}$ thick).

4. Low temperature absorption spectrum. - The absorption spectrum of $\mathrm{MnCl}_{2}$ at liquid helium temperature is plotted in figure 2 . We observe all the transitions reported by R. Pappalardo [10], from $18000 \mathrm{~cm}^{-1}$ to $31000 \mathrm{~cm}^{-1}$, and also the band at $30500 \mathrm{~cm}^{-1}$ already reported by R. Mehra [11] in their experiments at liquid nitrogen temperature.

The oscillator strengths for some of these bands are given in table I. They are of the same order of magnitude, although slightly smaller than those calculated by Pappalardo. As reported by Marzacco and Mc Clure [12], in all compounds with $\mathrm{Mn}^{2+}$ ions, all electronic transitions are spin forbidden, and also parity forbidden when the ion is at a site of inversion symmetry. Zero-phonon lines should therefore have very low intensity, $f \sim 10^{-9}-10^{-11}$, because they are only magnetic dipole allowed. These estimates are however not in agreement with the experimental values $\left(f \sim 10^{-6}-10^{-8}\right)$.

Two possible mechanisms can break the selection rules : one is the presence of odd parity lattice modes which perturb the inversion symmetry of $\mathrm{Mn}^{2+}$ sites, and can allow a mixing between levels of different parities. Besides this, the spin-orbit coupling can produce a mixing between levels of different spin multiplicity (for example, ${ }^{6} \mathrm{~A}_{1 \mathrm{~g}}\left({ }^{6} \mathrm{~S}\right)$ and ${ }^{4} \mathrm{~T}_{1 \mathrm{~g}}\left({ }^{4} \mathrm{P}\right)$ ). By these means an electric dipole transition can become partly allowed between states of different spin multiplicity and the same parity. The other one is the exciton-magnon interaction which should be quite independent of temperature. Lohr and Mc Clure [13], comparing the temperature dependence of the oscillator strength of several absorption bands in different manganese salts, have demonstrated that the latter mechanism is more important. In $\mathrm{MnCl}_{2}$, the absence of any strong temperature dependence of the oscillator strength is also consistent with the exciton-magnon process.

Consequently, the sharpest peaks that we observe (with $f \sim 10^{-7}$ ) cannot be pure zero-phonon magnetic-dipole transitions. The two peaks at $23557 \mathrm{~cm}^{-1}$ and $23792 \mathrm{~cm}^{-1}$ in the absorption band between 23000 and $24000 \mathrm{~cm}^{-1}$ are presumably a superposition of the magnetic dipole transition and of exciton-magnon transitions.

Following Pappalardo [14] and Goode [15], these two peaks can be attributed respectively to the ${ }^{6} \mathrm{~A}_{1}\left({ }^{6} \mathrm{~S}\right) \rightarrow{ }^{4} \mathrm{~A}_{1 \mathrm{~g}}\left({ }^{4} \mathrm{G}\right)$ and ${ }^{6} \mathrm{~A}_{1}\left({ }^{6} \mathrm{~S}\right) \rightarrow{ }^{4} \mathrm{E}_{\mathrm{g}}\left({ }^{4} \mathrm{G}\right)$ transitions. We observe exactly the same splitting as Pappalardo between these two transitions :

$$
\Delta \omega(1-4)=235 \mathrm{~cm}^{-1} \text {. }
$$

From the Koyde and Pryce discussion [16], the ${ }^{4} \mathrm{~A}_{1 \mathrm{~g}}$ level is lower than ${ }^{4} \mathrm{E}_{\mathrm{g}}$ in all cases where the covalency parameter $\varepsilon$ is smaller than 0.10 .

Nevertheless, some doubt exists about the position of the ${ }^{4} \mathrm{~A}_{1 \mathrm{~g}}$ level with respect to the ${ }^{4} \mathrm{E}_{\mathrm{g}}$ level. Other arguments suggest that the peak at $23557 \mathrm{~cm}^{-1}$ corresponds to the ${ }^{6} \mathrm{~A}_{1}\left({ }^{6} \mathrm{~S}\right) \rightarrow{ }^{4} \mathrm{E}_{\mathrm{g}}\left({ }^{4} \mathrm{G}\right)$ transition and the peak at $23792 \mathrm{~cm}^{-1}$ to the ${ }^{6} \mathrm{~A}_{1}\left({ }^{6} \mathrm{~S}\right) \rightarrow{ }^{4} \mathrm{~A}_{1 \mathrm{~g}}\left({ }^{4} \mathrm{G}\right)$ transition : the high resolution absorption spectrum of $\mathrm{MnBr}_{2}$ measured by Farge et al. [17] shows that the intense band at $23000 \mathrm{~cm}^{-1}$ and the ${ }^{4} \mathrm{E}_{\mathrm{g}}\left({ }^{4} \mathrm{D}\right)$ band at $27500 \mathrm{~cm}^{-1}$ have exactly the same shape, and so, that the former is perhaps also ${ }^{4} E_{g}$ in character. This assignment is in good agreement with the results of Schwartz et al. [18], who observed the ${ }^{6} \mathrm{~A}_{1 \mathrm{~g}} \rightarrow{ }^{4} \mathrm{~A}_{1 \mathrm{~g}}$ transition at higher energy than the ${ }^{6} \mathrm{~A}_{1 \mathrm{~g}} \rightarrow{ }^{4} \mathrm{E}_{\mathrm{g}}$ transition in $\mathrm{K}_{2} \mathrm{MnF}_{4}$ and $\mathrm{MnF}_{2}$.

Thirdly, in $\mathrm{MnCl}_{2}$, below the Néel temperature, the lineshape of the peak at $\omega=23557 \mathrm{~cm}^{-1}$ changes 

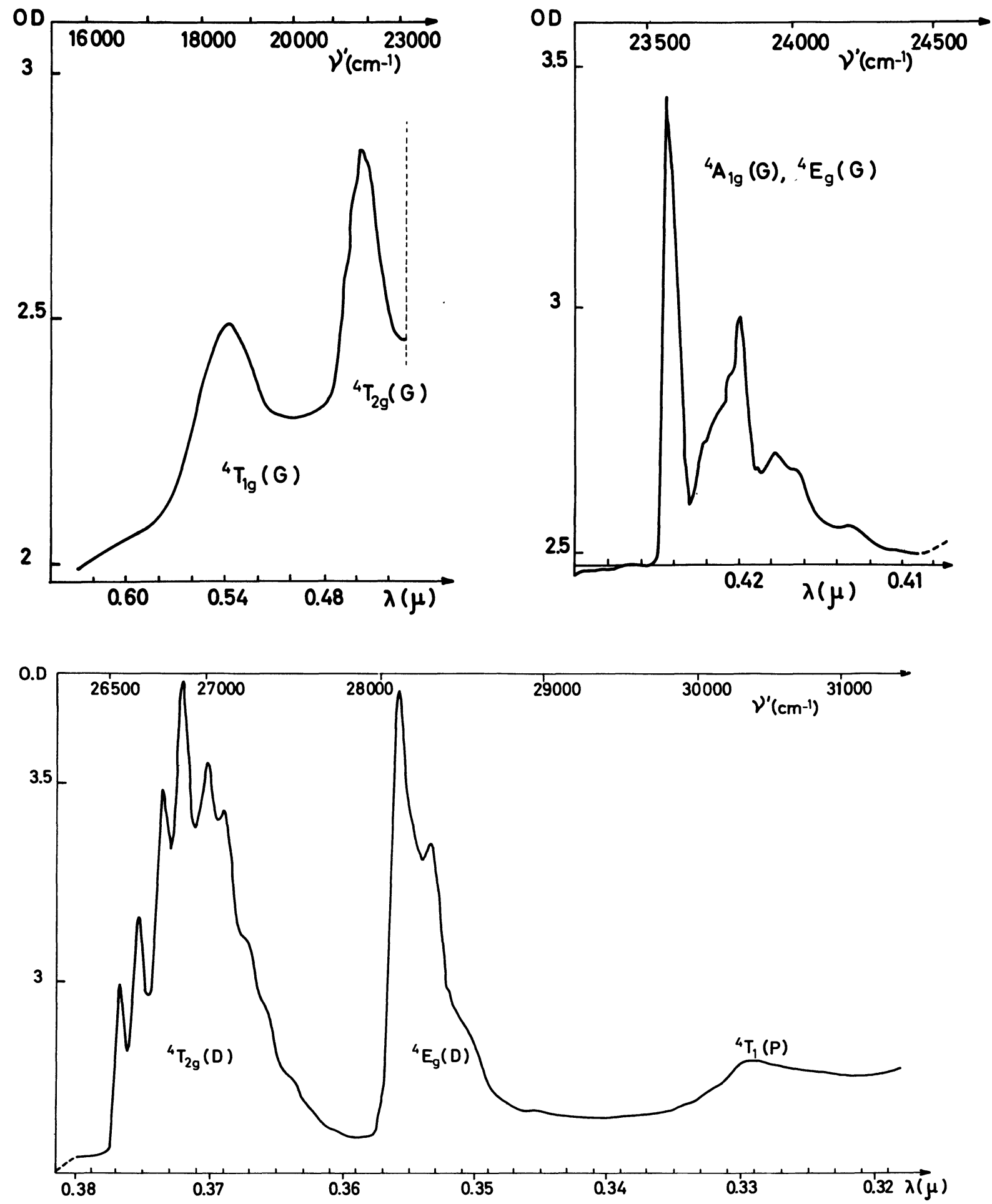

FIG. 2. - Absorption spectrum of $\mathrm{MnCl}_{2}$ single crystal at liquid helium temperature (spectral resolution 2 to $9 \mathrm{~cm}^{-1}$ ).

strongly and a shoulder appears. (It was not possible as it is for $\mathrm{MnBr}_{2}$, to detect a true splitting of the peak.) To this shoulder there corresponds a small peak in the MCD signal at a distance of $31 \mathrm{~cm}^{-1}$ from the main peak. Its intensity goes to zero at the Néel temperature. Although this band has the same behaviour as a cold band magnon sideband, it is very unlikely that magnon frequencies in $\mathrm{MnCl}_{2}$ are so high. As a result this band cannot be attributed to a sideband of the origin at $\omega=23557 \mathrm{~cm}^{-1}$. It has presumably another origin.

We can suppose that the splitting if the ${ }^{6} \mathrm{~A}_{1 \mathrm{~g}} \rightarrow{ }^{4} \mathrm{E}_{\mathrm{g}}$ transition is allowed by second order spin-orbit interaction, and that the small peak at $\Delta \omega=31 \mathrm{~cm}^{-1}$ is a cold band associated with the second component of the ${ }^{6} \mathrm{~A}_{1 \mathrm{~g}} \rightarrow{ }^{4} \mathrm{E}_{\mathrm{g}}$ transition. 
TABLE I

Observed Absorption Bands

\begin{tabular}{|c|c|c|c|c|}
\hline$\lambda(\mu)$ & $\omega\left(\mathrm{cm}^{-1}\right)$ & O.D. & Attribution & $f$ \\
\hline- & - & - & - & - \\
\hline 0.5420 & 18450 & 0.30 & ${ }^{6} \mathrm{~A}_{1}(\mathrm{~S}) \rightarrow{ }^{4} \mathrm{~T}_{1 \mathrm{~g}}(\mathrm{G})$ & $10^{-6}$ \\
\hline [1] 0.4570 & 21882 & 0.48 & $\rightarrow{ }^{4} \mathrm{~T}_{2 \mathrm{~g}}(\mathrm{G})$ & $1.2 \times 10^{-6}$ \\
\hline [1] 0.4245 & 23557 & 0.97 & $\rightarrow{ }^{4} \mathrm{E}_{\mathrm{g}}\left({ }^{4} \mathrm{G}\right)$ & $1.8 \times 10^{-7}$ \\
\hline [2] 0.4224 & 23674 & & & \\
\hline [3] 0.4214 & 23730 & & & \\
\hline [4] 0.4203 & 23792 & 0.51 & $\rightarrow{ }^{4} \mathrm{~A}_{1 \mathrm{~g}}\left({ }^{4} \mathrm{G}\right)$ & $1.4 \times 10^{-7}$ \\
\hline [5] 0.4180 & 23923 & & & \\
\hline [6] 0.4162 & 24026 & & & \\
\hline [7] 0.4133 & 24195 & & & \\
\hline 0.3768 & 26539 & 0.44 & & $0.93 \times 10^{-7}$ \\
\hline 0.3755 & 26631 & 0.60 & & $1.3 \times 10^{-7}$ \\
\hline 0.3736 & 26767 & & & \\
\hline 0.3722 & 26867 & 1.80 & & \\
\hline 0.3704 & 26998 & & $\rightarrow{ }^{4} \mathrm{~T}_{2 \mathrm{~g}}(\mathrm{D})$ & $3.3 \times 10^{-7}$ \\
\hline 0.3691 & 27093 & & & \\
\hline 0.3675 & 27211 & & & \\
\hline 0.3660 & 27322 & & & \\
\hline 0.3640 & 27472 & & & \\
\hline 0.3558 & 28106 & 1.11 & $\rightarrow{ }^{4} \mathrm{E}_{\mathrm{g}}(\mathrm{D})$ & $2.4 \times 10^{-7}$ \\
\hline 0.3535 & 28288 & & & \\
\hline 0.3513 & 28466 & & & \\
\hline 0.3485 & 28694 & & & \\
\hline 0.3290 & 30395 & 0.10 & $\rightarrow{ }^{4} \mathrm{~T}_{1}(\mathrm{P})$ & \\
\hline
\end{tabular}

The main peak at $23557 \mathrm{~cm}^{-1}$ would be the other component of the ${ }^{6} \mathrm{~A}_{1 \mathrm{~g}} \rightarrow{ }^{4} \mathrm{E}_{\mathrm{g}}$ transition, with associated magnon sidebands.

Besides these two peaks, there are several bands corresponding to a vibronic structure. The energy separation between the main peaks and these bands are

$$
\begin{gathered}
\omega_{(1.2)}=117 \mathrm{~cm}^{-1}, \omega_{(1 ; 3)}=173 \mathrm{~cm}^{-1}, \\
\omega_{(4-5)}=131 \mathrm{~cm}^{-1} \text { and } \omega_{(4-6)}=234 \mathrm{~cm}^{-1} .
\end{gathered}
$$

These frequencies correspond roughly to the phonon frequencies deduced from infra-red and Raman measurements. These spectra have been measured by Lockwood [3], who found that the Raman active phonon modes are the $A_{1 \mathrm{~g}}$ mode at $234.5 \mathrm{~cm}^{-1}$ and the $E_{\mathrm{g}}$ at $144 \mathrm{~cm}^{-1}$, the IR active modes being the $A_{2 u}$ at $180 \mathrm{~cm}^{-1}$ and $E_{u}$ at $230 \mathrm{~cm}^{-1}$. Such a vibronic structure is also observed in the ${ }^{4} \mathrm{~T}_{2 \mathrm{~g}}(\mathrm{D})$ and ${ }^{4} \mathrm{E}_{\mathrm{g}}(\mathrm{D})$ levels.

5. Magnetic circular dichroism of the band at $\omega=23557 \mathrm{~cm}^{-1}$. - (Light beam and applied magnetic field parallel to the $\mathbf{c}$ axis of the crystal.)

5.1 CORRELATION BETWEen THE MCD Signal AND THE MAGNETIZATION. - In our experiments in $\mathrm{MnCl}_{2}$, it was not possible to use a magnetic field parallel to the direction of the magnetic moments. We have therefore performed two kinds of experiments : first, the MCD measurements were done with light and applied magnetic field perpendicular to the magnetic moments. Then, the same measurements were done with the $c$ axis of the sample at $45^{\circ}$ with the light beam, to have a component of the applied magnetic field $\mathbf{H}$ parallel to the direction of the spins. With this geometry, we hoped to obtain more spectacular results, but the experimental data are much more complex than in the first case, as we shall see.

In the first geometry, (spins in the hexagonal planes, light and $\mathbf{H}$ parallel to the $\mathbf{c}$ axis), we can calculate, in the simplest case, the expected intensity of the MCD signal. We assume a pure electronic, magnetic dipole transition between the ${ }^{6} A_{1}\left({ }^{6} S\right)$ to the ${ }^{4} A_{1}\left({ }^{4} G\right)$, $\left.{ }^{4} \mathrm{E}_{\mathrm{g}}{ }^{4} \mathrm{G}\right)$ levels, because it has been shown by Schwartz et al. [18] that, in the case of $\mathrm{MnF}_{2}$ and $\mathrm{K}_{2} \mathrm{MnF}_{4}$, the more important signals of MCD come from the purely electronic, magnetic dipole transitions. The magnon sidebands, often electric dipole in character, give smaller contributions to the MCD, because the spin is conserved in these transitions.

First, we assume that we have an antiferromagnetic system with two sublattices, the magnetic moments being aligned in the $\mathbf{z}$ direction and the local exchange 
field on each sublattice being $H_{z}$ and $-H_{z}$. The applied magnetic field $H_{x}$, in the direction $\mathbf{x}$ perpendicular to $\mathbf{z}$, acts as a first order perturbation on the energy levels of the $\mathrm{Mn}^{2+}$ ions, already split by $H_{z}\left({ }^{1}\right)$. It therefore produces a mixing between the Zeeman sublevels and we have to calculate the matrix elements of the operator $g \mu_{\mathrm{B}} H_{x} S_{x}$ on the spin wave functions of the ${ }^{6} \mathrm{~A}_{1}\left({ }^{6} \mathrm{~S}\right)$ level.

In this temperature range, $(T<2 \mathrm{~K})$, we assume that only the lower sublevel of the ground state, for each sublattice, is populated and that the exchange field $H_{z}$ is the same for the ground and the excited state. In this case, we have found that the perturbation due to the applied field $H_{x}$ on the excited states ${ }^{4} \mathrm{~A}_{1 \mathrm{~g}}$ and ${ }^{4} E_{g}$, has negligible effect on the calculation of the MCD intensity, and we consider only this perturbation on the ground level.

The calculation of the new perturbated wave function for the ground level for sublattice A gives :

$$
\psi_{0}^{\mathrm{A}}=\alpha\left|{ }^{6} \mathrm{~A}_{1 \mathrm{~g}},-\frac{5}{2}\right\rangle+\frac{\sqrt{5}}{2} \frac{H_{x}}{H_{z}}\left|{ }^{6} \mathrm{~A}_{1 \mathrm{~g}},-\frac{3}{2}\right\rangle
$$

with

$$
\alpha=\left[1-\frac{5}{4}\left(\frac{H_{x}}{H_{z}}\right)^{2}\right]^{1 / 2} \simeq 1 .
$$

Taking into account the small mixing of various ${ }^{4} \mathrm{~T}_{1}$ states with the ground state, via spin-orbit coupling, gives :

$$
\begin{aligned}
\psi_{0}^{\prime \mathrm{A}} & =\alpha^{\prime}\left|{ }^{6} \mathrm{~A}_{1 \mathrm{~g}},-\frac{5}{2}\right\rangle+\frac{\sqrt{5}}{2} \frac{H_{x}}{H_{z}} \beta^{\prime}\left|{ }^{6} \mathrm{~A}_{1 \mathrm{~g}},-\frac{3}{2}\right\rangle+ \\
& +A_{\mathrm{m}}\left|{ }^{4} \mathrm{~T}_{1},-1,-\frac{3}{2}\right\rangle+\frac{\sqrt{5}}{2} \frac{H_{x}}{H_{z}} A_{\mathrm{n}}\left|{ }^{4} \mathrm{~T}_{1},-1,-\frac{1}{2}\right\rangle \\
& +\frac{\sqrt{5}}{2} \frac{H_{x}}{H_{z}} A_{\mathrm{p}}\left|{ }^{4} \mathrm{~T}_{1}, 0,-\frac{3}{2}\right\rangle .
\end{aligned}
$$

Only the three last terms are efficient in the transition intensity. $A_{\mathrm{m}}, A_{\mathrm{n}}, A_{\mathrm{p}}$ are coefficients of mixture. The wave functions for the ${ }^{4} \mathrm{~A}_{1 \mathrm{~g}}$ level (sublattice A) are :

$$
\begin{aligned}
& \psi_{1}^{\mathrm{A}}=\left|{ }^{4} \mathrm{~A}_{1 \mathrm{~g}},-\frac{3}{2}\right\rangle \\
& \psi_{2}^{\mathrm{A}}=\left|{ }^{4} \mathrm{~A}_{1 \mathrm{~g}},-\frac{1}{2}\right\rangle .
\end{aligned}
$$

The magnetic dipole operator is $\mu(L+2 S)$. The transition probabilities for the right $\left(I_{\mathrm{R}}\right)$ and left $\left(I_{\mathrm{L}}\right)$ circularly polarized absorptions are deduced by application of the $L_{z} \pm i L_{y}$ operator between the

( $\left.{ }^{1}\right)$ This approximation is valid when $H_{x} \ll H_{z}, \quad\left(H_{z}\right.$ is presumably of the order of $10 \mathrm{kG}$ in phase II). For the highest values of the applied field $H_{x}$, the effects will be no more linear. ground and excited state. Finally we get for the MCD signal intensity of the ${ }^{6} \mathrm{~A}_{1 \mathrm{~g}} \rightarrow{ }^{4} \mathrm{~A}_{1 \mathrm{~g}}$ transition :

$$
S=\frac{I_{\mathrm{R}}-I_{\mathrm{L}}}{I_{\mathrm{R}}+I_{\mathrm{L}}}=-\frac{2 \sqrt{5}}{3} \frac{H_{x}}{H_{z}} A_{\mathrm{p}} \cdot A_{\mathrm{m}} .
$$

The wave functions of the ${ }^{4} \mathrm{E}_{\mathbf{g}}$ level (sublattice $\mathrm{A}$ ) are :

$$
\begin{aligned}
& \psi_{3}^{\mathbf{A}}=\left|{ }^{4} \mathrm{E}_{\theta},-\frac{3}{2}\right\rangle \quad \psi_{5}^{\mathbf{A}}=\left|{ }^{4} \mathrm{E}_{\varepsilon},-\frac{3}{2}\right\rangle \\
& \psi_{4}^{\mathbf{A}}=\left|{ }^{4} \mathrm{E}_{\theta},-\frac{1}{2}\right\rangle \quad \psi_{6}^{\mathbf{A}}=\left|{ }^{4} \mathrm{E}_{\varepsilon},-\frac{1}{2}\right\rangle .
\end{aligned}
$$

The same calculation gives the MCD intensity

$$
S^{\prime}=\frac{2 \sqrt{5}}{3} \frac{H_{x}}{H_{z}} A_{\mathrm{p}} \cdot A_{\mathrm{m}}
$$

for the ${ }^{6} \mathrm{~A}_{1 \mathrm{~g}} \rightarrow{ }^{4} \mathrm{E}_{\mathrm{g}}$ transition. We see that $S$ and $S^{\prime}$ have the same value and opposite sign. This result is in good agreement with experiment : we observe that the MCD signal given by the band at $\omega=23792 \mathrm{~cm}^{-1}$ has opposite sign and about the same value as the MCD of the band at $\omega=23557 \mathrm{~cm}^{-1}$.

It is easy to see that the perpendicular magnetization is also proportionnal to $H_{x} / H_{z}$ and so, there is a linear correlation between the MCD signal and the magnetization.

These simple calculations enable us to predict that :

1. - there is no shift in energy between the fundamental lower sublevels corresponding to the two sublattices and we therefore expect no first moment change in the MCD signal;

2. - the contributions of the two sublattices to the MCD signal have the same sign and the same intensity, so that we expect a signal mainly due to a zeroth moment change (i.e. : a single peak), and its - sign should be reversed when the applied magnetic field changes sign.

5.2 EXPERIMENTAL RESUlts. - 5.2.1 Circular dichroism in zero field. - A circular dichroic signal, in zero applied magnetic field, is observed for the band at $\omega=23557 . \mathrm{cm}^{-1}$. This signal appears below $1.96 \mathrm{~K}$ (i.e. at the first phase transition) and diminishes below $1.81 \mathrm{~K}$, at the second phase transition. Figure 3 shows this C.D. signal as a function of temperature

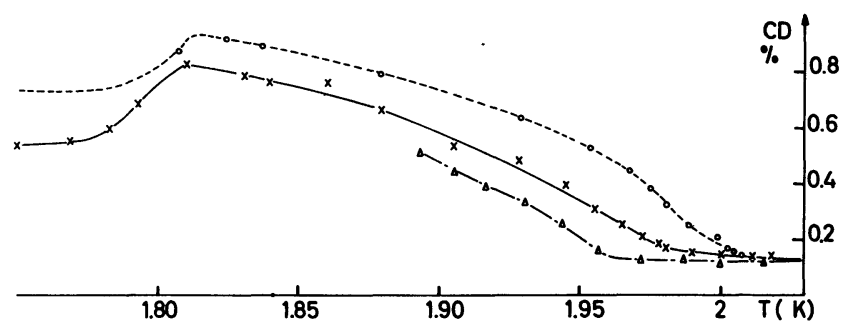

FIG. 3. - Intensity of the circular dichroism in zero field, for three different samples. 
for three different samples. Its intensity, and also the temperature at which it appears, are slightly different for the three crystals.

A change can be observed in the C.D. signal for the same sample, with different focalization of the light beam. With a broad beam, the C.D. signal was zero at all temperatures, even in the phase $I$.

Such a zero field C.D. has been already observed in the magnon sideband associated with the ${ }^{6} \mathrm{~A}_{1 \mathrm{~g}} \rightarrow{ }^{4} \mathrm{~A}_{1 \mathrm{~g}}$ transition in $\mathrm{MnF}_{2}$, by Wong et al. $[19,20]$ and has the same, non-differential shape as in the case of $\mathrm{MnCl}_{2}$. The origin of this C.D. signal is not completely understood, but was attributed by these authors to a stress induced in the sample during magnetic ordering.

The effect of the size of the beam on the zero field C.D. signal indicates that this signal is due to the domains, in the phase I. Following the model of Wilkinson et al., there are three types of magnetic domains, at $120^{\circ}$ from each other. Perhaps, with a broad light beam, the effect of the three types of domains can counteract, and the C.D. signal is zero.

Two hypotheses can explain this zero field C.D. signal. First, the magnetostriction in a domain lowers the symmetry of the crystal, which becomes birefringent. This effect can induce a small linear dichroism, which can be detected at the same frequency (see section 6), and wrongly interpreted as a circular dichroism.

Another possible mechanism is that the phase I is not a true antiferromagnetic phase, but a weak ferromagnet, with domains.

In such a model, with a component of the spontaneous magnetization in the c direction, the ${ }^{6} \mathrm{~A}_{1}(\mathrm{~S})$ ground level can be split and a circular dichroism can be induced. If the magnetization has a different direction in different domains, it is possible to null the signal when the part of the sample which is illuminated contains many domains.

However, we did not succeed in observing the light scattered by the magnetic domains in $\mathrm{MnCl}_{2}$, using a laser as we have done in the case of $\mathrm{FeCl}_{2}$ [5]. This negative result suggests that the domains are too large or that the coupling between the light and the crystal is too small as would be the case if a small ferromagnetism is present in the phase I.

Whatever was the size of the light beam on the sample, the MCD signal (in applied magnetic field $\neq 0$ ) was unaffected and, in the following, we can say (as Wong et al.) that the origin of C.D. and MCD are quite different and thus the effects are independent and simply additive.

5.2.2 Magnetic circular dichroism. - As expected, the MCD signal has mainly the shape of a single peak, centered at $\omega=23557 \mathrm{~cm}^{-1}$, and the sign of the signal changes when the applied magnetic field is reversed. A second small peak appears, at $31 \mathrm{~cm}^{-1}$ above the main peak, when the first transition tempe- rature is reached. The integrated area of the main peak (corrected for the zero field C.D.) is plotted in figure 4 as a function of temperature, for different values of the applied field.

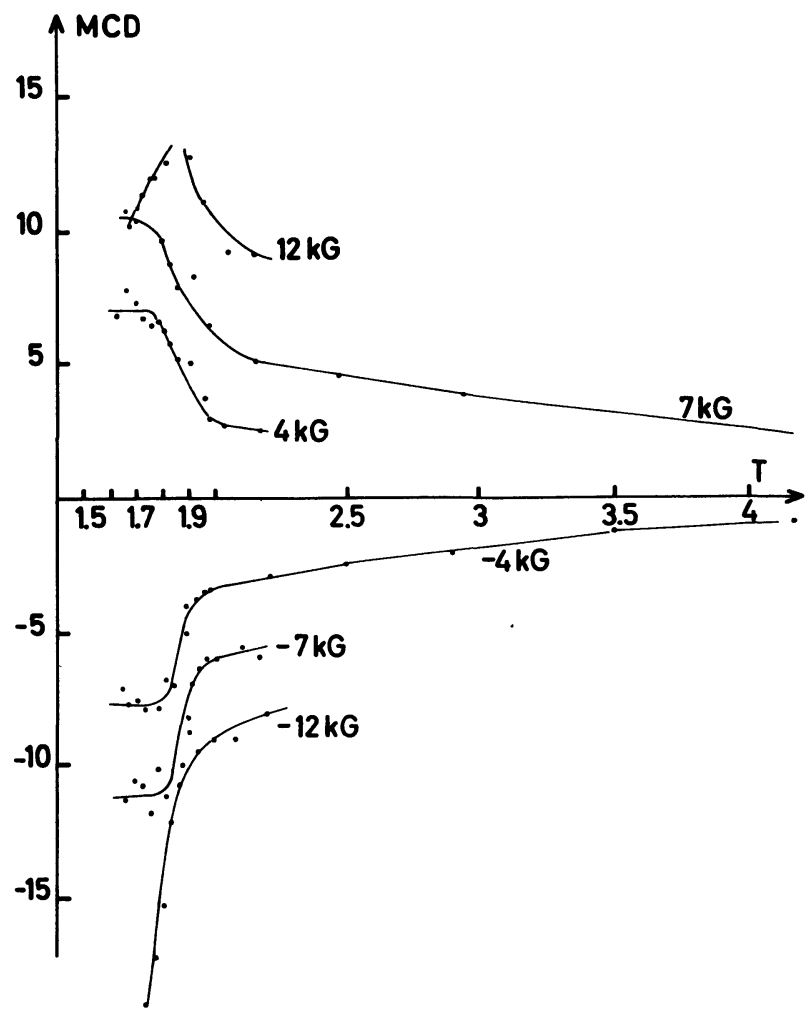

FIG. $4 a$. - Intensity of the MCD signal visus temperature. Sample with $\mathrm{c}$ axis $/ /$ to the light beam.

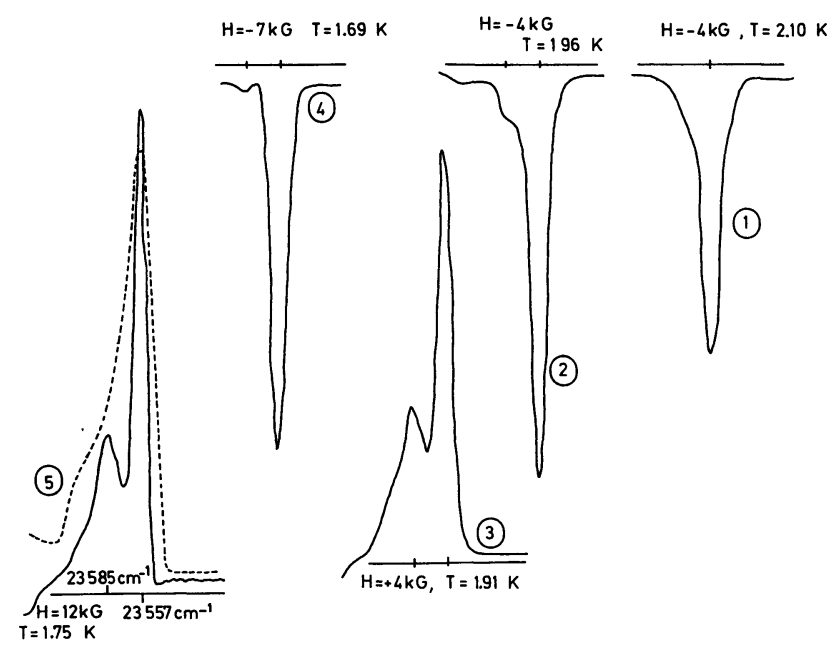

FIG. 4b. - Experimental MCD signal at various temperatures and fields. (The intensity of (1), (2), (3) is multiplied by 2,5 ).

This MCD signal, or perpendicular magnetization, deviates from the Curie law below $4.18 \mathrm{~K}$ in the paramagnetic phase. Below the first transition temperature in phase $I$, the magnetization increases with a larger slope when the temperature decreases. For the two smallest values of the applied field $( \pm 4 \mathrm{kG}$, $\pm 7 \mathrm{kG}$ ), the MCD signal saturates below $1.80 \mathrm{~K}$ 
when the transition from phase I to phase II is reached. In phase II, the magnetization is nearly constant with the temperature at constant field, indicating that the perpendicular susceptibility is constant, as is expected in an antiferromagnetic material. This is confirmed by the fact that in phase II, the ratio of the MCD intensity and the applied magnetic field ( $\pm 4 \mathrm{kG}, \pm 7 \mathrm{kG})$ is a constant, proportional to the transverse susceptibility.

In figure 5, the MCD signal is plotted (with the same units as in figure 4) at constant temperature, in phase $\mathrm{I},(T=1.89 \mathrm{~K})$, versus the applied field. The magnetization grows linearly with field and then saturates when the field is high enough to reach the antiferromagnetic-paramagnetic phase transition. It is a classical curve of perpendicular magnetization in an antiferromagnetic material. But the absence of symmetry of this curve, when the field is reversed, is not understood in a model of an ordered phase. The slope of this curve is proportional to the differential perpendicular susceptibility $\mathrm{d} M / \mathrm{d} H$. From such curves plotted at different temperatures in phase $\mathrm{I}$, we have verified that $\mathrm{d} M / \mathrm{d} H$ is not constant (as reported in the Watanabe experiments), but increases when the temperature decreases (as mentionned by Murray and Roberts). This property appears also in figure $4 a$, on the plots at constant field.

There was no evidence of hysteresis in the linear part of the curve. We only observed a small hysteresis when the MCD saturates.

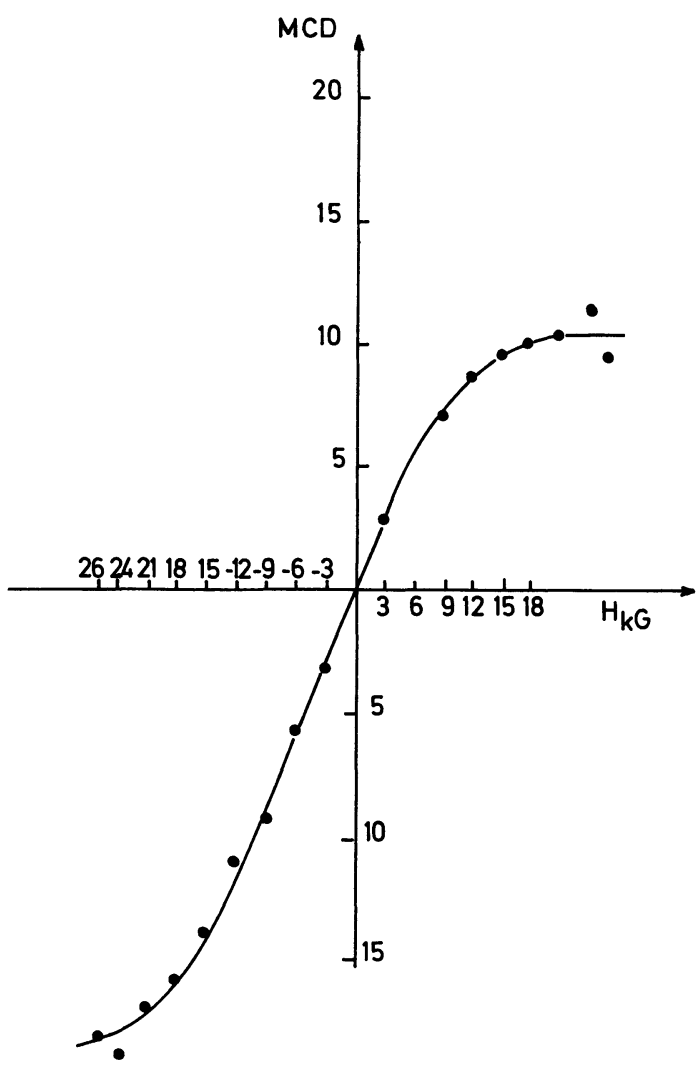

FIG. 5. - MCD versus field at constant temperature in phase I $(T=1.89 \mathrm{~K})$.
At lower temperature $(T=1.65 \mathrm{~K})$, the plot of MCD versus field at constant temperature in function of the applied field is presented in figure 6 and not so well understood, as the isothermal plot at $1.89 \mathrm{~K}$. We have not yet an explanation for the strong lowering of the dichroism signal when the first transition (phase I $\rightarrow$ paramagnetic phase) is reached, and for the lack of symmetry when the field is reversed. A small hysteresis is observed in this diagram in the range of field corresponding to phase II.

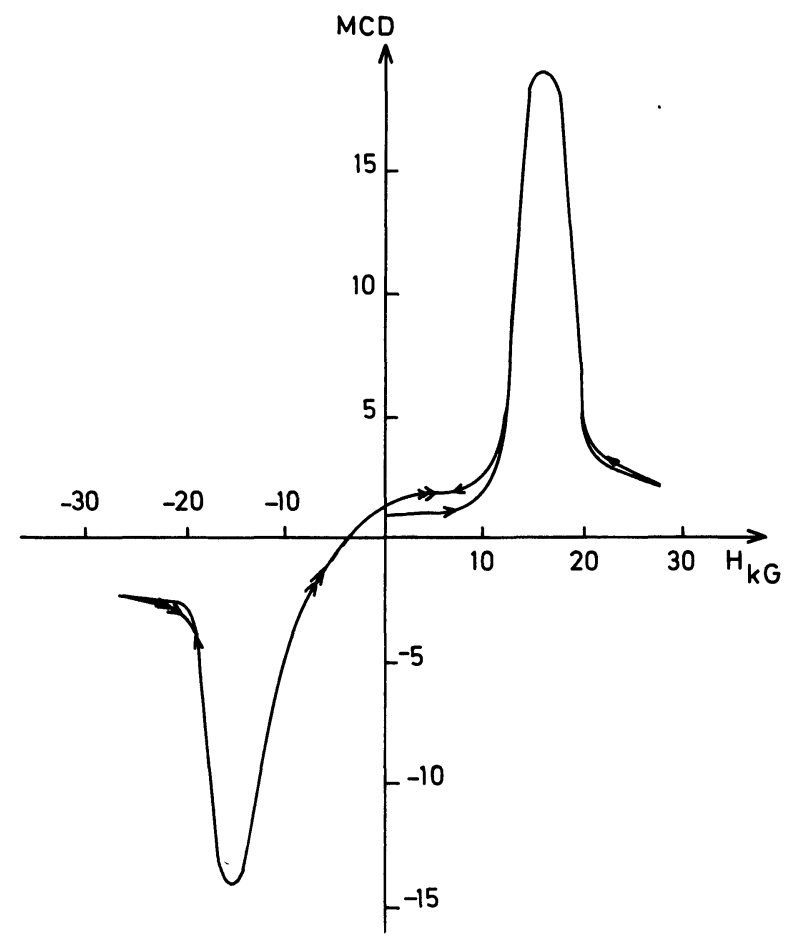

Fig. 6. - MCD signal versus field at $1.65 \mathrm{~K}$.

5.2.3 Magnetic phase diagram. - The change of the MCD signal at the transition temperatures is used to plot the magnetic phase diagram of $\mathrm{MnCl}_{2}$, down to $1.65 \mathrm{~K}$ in temperature and up to $20 \mathrm{kG}$ in applied magnetic field (Fig. 7). Careful measurements of the first transition temperature (between the paramagnetic phase and the AFM phase I) were performed, with small applied fields, in order to check whether or not there is a coincidence between

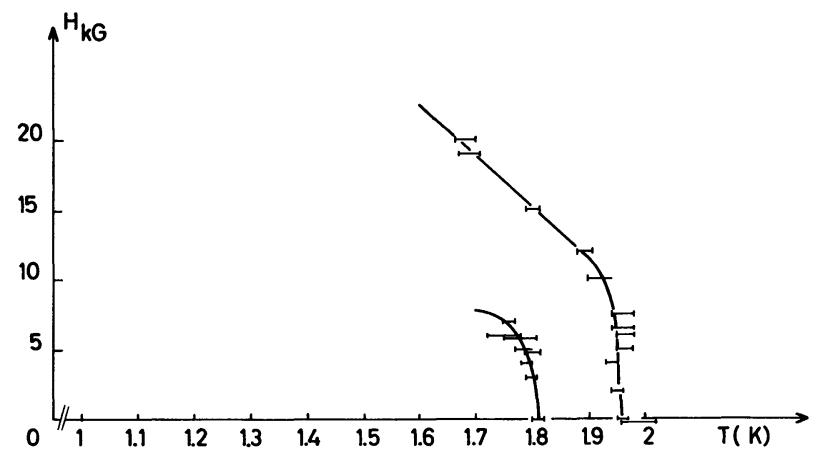

Fig. 7. - Magnetic phase diagram. 
our results and the phase diagram plotted by Butera and Giauque with heat-capacity measurements. We have not observed, as they did, a raising of the transition temperature with the applied field, and the transition temperature seems to be constant in the range 0-8 kG.

We report a transition temperature

$$
T_{1}=1.96 \pm 0.05 \mathrm{~K}
$$

in agreement with previous measurements, and a second transition temperature (from phase $I$ to phase II) $T_{2}=1.81 \pm 0.05 \mathrm{~K}$ in zero applied field.

6. Light beam and magnetic field at $45^{\circ}$ to the $\mathrm{c}$ axis of the sample. - In this geometry, the dichroic signal detected at the frequency of the electro-optic modulator is expected to be due to three contributions.

First, one component of the applied magnetic field is parallel to the direction of the magnetic moments of the sample. It induces a different splitting for the Zeeman sublevels of ${ }^{6} \mathrm{~A}_{1}\left({ }^{6} \mathrm{~S}\right)$ for the two sublattices. Its effect on the MCD is a differential signal corresponding to a first moment change for the $\sigma^{+}$and $\sigma^{-}$ components of the absorption band.

Second, another component of the applied magnetic field is still directed parallel to the c axis, and so, gives a peak in the MCD corresponding to a zeroth moment change, as we have seen in the previous geometry. The sign of this part of the signal must change when the applied field is reversed.

Third, with this geometry, the crystal is no longer uniaxial, but biaxial and can behave as a retardation plate. It can transform partly or totally a circular dichroism into a linear one, and reciprocally. Then, at the frequency of vibration of the modulator, one could detect not only a circular dichroism but also a linear dichroism, induced for example by the magnetostriction.

With this geometry, the detected signal is mainly due to the third contribution for the two following reasons :

- first, its sign remains the same when the field is reversed;

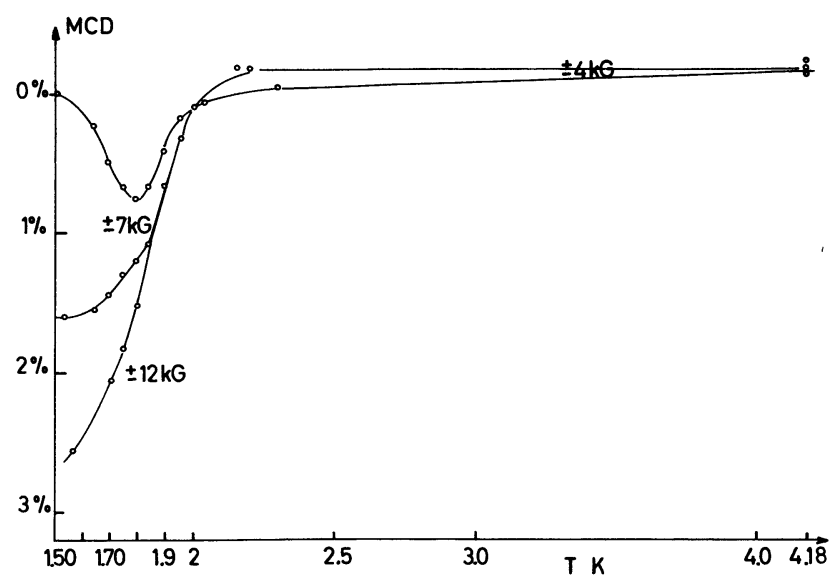

FIG. 8. - Sample inclinated at $45 \%$ with regard to the light beam. MCD (or LD) signal intensity in function of temperature.

LE JOURNAL DE PHYSIQUE. - T. 37, No 5, MAI 1976
- secondly, it corresponds essentially to a zeroth moment change (Fig. 9). In order to check this interpretation, we have measured directly the linear dichroism induced by the crystal and found that it is identical to the presumed circular one.

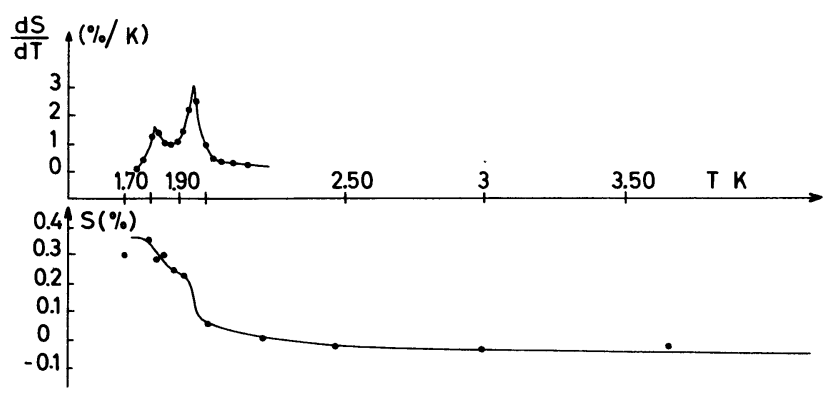

FIG. 9. - Sample inclinated at $45 \%$ with regard to the light beam. CD (or LD) signal in zero field and first derivative.

In this geometry, the strongest contribution to the observed signal is therefore a linear dichroism which probably arises from the magnetostriction which reduces the local symmetry and acts as a distortion. Because this distortion is very small, we can assume that the linear dichroism is proportional to the magnetostriction. This effect on the imaginary part of the refractive index is exactly the same as on the linear magnetic birefringence which is coming from the real part of the refractive index. Following the work of Jahn et al. [4] on antiferromagnets and of Kleeman et al. [4] on $\mathrm{K}_{2} \mathrm{CuF}_{4}$ and $\mathrm{K}_{2} \mathrm{NiF}_{4}$, this magnetostriction is proportional to the magnetic energy $U_{\mathrm{m}}$. Thus, the first derivative of the linear dichroism versus temperature is proportional to the magnetic heat capacity $C_{\mathrm{m}}$ of the crystal. This dichroism and its derivative versus temperature in zero field are plotted in figure 9. The derivative clearly shows two peaks, analogous to those measured by Murray et al.

The temperatures of the two peaks are in good agreement with the transition temperatures previously reported : $T_{1}=1.96 \mathrm{~K}, T_{2}=1.81 \mathrm{~K}$. From this curve it is clear that the peaks are not as high as in a three dimensional magnetic compound. But Murray et al. observed a tail in the curve above the Néel point smaller than in a perfect two dimensional material, indicating that short range magnetic order remains above the transition. This tail is not evident in our experiment, probably because of the complex nature of the signal.

The optical measurements of either magnetic birefringence or linear dichroism have in general a great advantage. They allow one to monitor the magnetic specific heat directly, without substracting the lattice specific heat, which can introduce an important error. (However, in the case of $\mathrm{MnCl}_{2}, T_{\mathrm{N}}$ is so low that the phonon specific heat is negligible.)

7. Discussion and conclusion. - In our experiments, we have obtained by MCD techniques the two tran- 
sition temperatures of $\mathrm{MnCl}_{2}\left(T_{1}=1.96 \mathrm{~K}\right.$ and $T_{2}=1.81 \mathrm{~K}$ ) which were found to be in agreement with preceding authors, and we have measured the magnetic phase diagram up to $20 \mathrm{kG}$ and down to $1.6 \mathrm{~K}$.

From our results, it appears that the low temperature phase corresponds to a three dimensional antiferromagnetic order with the magnetic moments lying in the hexagonal planes : the perpendicular magnetization and the perpendicular susceptibility are constant when the temperature decreases.

The nature of the intermediate phase is not yet clear. Several of its properties cannot be explained by a model of an ordered antiferromagnetic phase as proposed by Wilkinson et al. : the perpendicular susceptibility increases when the temperature decreases. The magnetization curves $M(H)$ are not symmetric with respect to the origin.

The origin of the C.D. in zero field is not clear, and can come as well from the magnetostriction during the magnetic ordering as from a weak ferromagnetism. The presence of magnetic domains in this phase was pointed out by the neutron diffraction experiments, and is undoubtly responsible for the change in the zero field C.D. signal with the size of the light beam on the sample.

Butera and Giauque think that there are not two different antiferromagnetic structures in $\mathrm{MnCl}_{2}$ involved in the transitions indicated by the heat capacity maxima, but that these maxima indicate gradual ordering in a complex system. Murray's results show that the transitions are not of the first order, and probably not of the second order also. In the temperature range of the existence of the phase I (1.81-1.96 K), a great amount of magnetic order does not exist, because the major part of the maximum spin entropy is still left (Butera and Giauque). The $\mathrm{MnCl}_{2}$ phase I seems to behave like a partly ordered system. Perhaps only a two dimensional order exists which becomes more and more three dimensional, as the temperature decreases but, in this model, it is not clear that magnetic domains can exist. Another hypothesis is that ferromagnetic order begins to exist, in the direction at $57^{\circ}$ to the crystalline c axis; in this geometry, the formation of domains can be more easily favored.

Further experiments, in particular with the use of neutron diffraction, are still necessary to determine its magnetic structure with a better precision. In the case of $\mathrm{MnBr}_{2}$, we will draw the same conclusions as for $\mathrm{MnCl}_{2}$. In $\mathrm{MnBr}_{2}$, a lot of effects are more pronounced but are of exactly the same nature as in $\mathrm{MnCl}_{2}$.

In conclusion, we wish also to underline the advantages of MCD techniques in this kind of study. MCD permits to separate some optical transitions not resolved in the absorption spectrum. The correlation between MCD and perpendicular magnetization gives a new tool to study the differential susceptibility. Moreover, the changes in MCD signal at the transition temperatures can be used to plot the magnetic phase diagram. Finally, we have shown that the linear dichroic signal, induced by the magnetostriction, can be used to derive the magnetic heat capacity.

Acknowledgments. - The authors thank D. Raoux and J. Ferré for helpful discussions.

\section{References}

[1] Cf for example :

Sell, D. D., Green, R. L. and White, R. M., Phys. Rev. 158 (1967) 489.

SChnatterly, S., Fontana, M., J. Physique 33 (1972) 691.

[2] Griffin, J., Schnatterly, S., Phys. Rev. Lett. 33 (1974) 1576.

[3] Lockwood, D. J., J. Opt. Soc. Am. 63 (1973) 374.

Lehmann, W. and Weber, R., Phys. Lett. 45A (1973) 33.

Mac Farlane, R. M. and Luntz, A. C., Phys. Rev. Lett. 31 (1973) 832.

Meltzer, R. S., Chen, M. Y., McClure, D. S., LowePariseau, M., Phys. Rev. Lett. 21 (1968) 913.

Greene, R. L., Sell, D. D., Yen, W. M., Shawlow, A. L., White, R. M., Phys. Rev. Lett. 15 (1965) 656.

[4] Jahn, I. R. and Bittermann, K., Solid State Commun. 13 (1973) 1897.

KleemanN, W. and Farge, Y., J. Physique Lett. 35 (1974) L-135.

[5] Griffin, J., Schnatterly, S., Farge, Y., RéGis, M., Fontana, M., Phys. Rev. B 10 (1974) 1960.

Wong, Y. H., Scarpace, F. L., Pfeifer, C. D., Yen, W. M., Phys. Rev. B 9 (1974) 3086.

[6] Murray, R. B., Phys. Rev. 100 (1955) 1071.

Butera, R. A. and Giauque, W. F., J. Chem. Phys. 40 (1964) 2379.

Murray, R. B., Phys. Rev. 128 (1962) 1570.

[7] Murray, R. B., RoberTs, L. D., Phys. Rev. 100 (1955) 1067.
Giauque, W. F. et al., J. Chem. Phys. 42 (1965) 9.

Watanabe, T., J. Phys. Soc. Japan 16 (1961) 1131.

[8] Wilkinson, M. K., Cable, J. W., Wollan, E. O., KoehlerORNL, W. C., Oak Ridge Nat. Lab. Rep. 2430 (1958) 65. 2501 (1958) 37.

[9] Jasperson, S. and Schnatterly, S., Rev. Sci. Instrum. 40 (1969) 761.

[10] Rappalardo, R., J. Chem. Phys. 31 (1959) 1050.

[11] Mehra, A., J. Chem. Phys. 48 (1968) 1871.

[12] Marzacco, C. J. and Mac Clure, D. S., Magneto optical effects Symposium of the Faraday Society, No 3 (1969) p. 106.

[13] Lohr, L. L. and Mac Clure, D. S., J. Chem. Phys. 49 (1968) 3516.

[14] Pappalardo, R., J. Chem. Phys. 33 (1960) 613.

[15] Goode, D. H., J. Chem. Phys. 43 (1965) 2830.

[16] Koide, S., Pryce, M. H. L., Phil. Mag. 3 (1958) 607.

[17] Farge, Y., Regis, M., Royce, B., J. Physique 37 (1976) 637.

[18] Schwartz, R. W., SPencer, J. A., Yeakel, W. C., Schatz, P. N., Maisch, W. G., J. Chem. Phys. 60 (1974) 2598.

[19] Wong, Y. H., Scarpace, F. L., Pfeifer, C. D., Yen, W. M., Phys. Rev. B (1974) 3086.

[20] Wong, Y. H., SCARPaCe, F. L., Chen, M. Y. and Yen, W. M., AIP Conf. Proceedings, No 5, part. I, Magnetism and Magnetic Materials (Graham and Rhynes, Ed.) 1971. 